\title{
MODULAR LIE REPRESENTATIONS OF FINITE GROUPS
}

\author{
R. M. BRYANT \\ (Received 17 January 2003; revised 21 July 2003) \\ Communicated by E. A. O'Brien
}

\begin{abstract}
Let $K$ be a field of prime characteristic $p$ and let $G$ be a finite group with a Sylow $p$-subgroup of order $p$. For any finite-dimensional $K G$-module $V$ and any positive integer $n$, let $L^{n}(V)$ denote the $n$th homogeneous component of the free Lie $K$-algebra generated by (a basis of) $V$. Then $L^{n}(V)$ can be considered as a $K G$-module, called the $n$th Lie power of $V$. The main result of the paper is a formula which describes the module structure of $L^{n}(V)$ up to isomorphism.
\end{abstract}

2000 Mathematics subject classification: primary 17B01; secondary $20 \mathrm{C} 20$.

\section{Introduction}

Let $G$ be a group and $K$ a field. For any finite-dimensional $K G$-module $V$, let $L(V)$ be the free Lie algebra over $K$ freely generated by any $K$-basis of $V$. Then $L(V)$ may be regarded as a $K G$-module on which each element of $G$ acts as a Lie algebra automorphism. Furthermore, each homogeneous component $L^{n}(V)$ is a finite-dimensional submodule, called the $n$th Lie power of $V$.

In this paper we consider the case where $K$ has prime characteristic $p$ and $G$ is a finite group with a Sylow $p$-subgroup of order $p$. We give a formula which describes $L^{n}(V)$ up to isomorphism for every finite-dimensional $K G$-module $V$. The formula has a strong resemblance to Brandt's character formula in characteristic zero [4], but the proof is much deeper.

In [6] a similar (but slightly simpler) formula was obtained for the case where $G$ is cyclic of order $p$. The present paper builds on [6] and earlier papers by the author, Kovács and Stöhr: particularly [9]. The results cover the symmetric group of degree $r$

(C) 2004 Australian Mathematical Society $1446-7887 / 04 \$ A 2.00+0.00$ 
with $p \leq r<2 p$ and the general linear group $\mathrm{GL}(2, p)$. These cases were studied in $[8,17,10]$, but closed formulae could not be given there except in special cases. We shall examine some of the connections between these papers and the present paper in Section 7 below.

For any group $G$ and any field $K$, we consider the Green ring (representation ring) $R_{K G}$. This is the ring formed from isomorphism classes of finite-dimensional $K G$-modules, with addition and multiplication coming from direct sums and tensor products, respectively. For any finite-dimensional $K G$-module $V$ we also write $V$ for the corresponding element of $R_{K G}$. Thus $V^{n}$ corresponds to the $n$th tensor power of $V$, and $L^{n}(V)$ may also be regarded as an element of $R_{K G}$.

In [5] it is shown that there exist $\mathbb{Z}$-linear functions $\Phi_{K G}^{1}, \Phi_{K G}^{2}, \ldots$ on $R_{K G}$ such that, for every finite-dimensional $K G$-module $V$ and every positive integer $n$,

$$
L^{n}(V)=\frac{1}{n} \sum_{d \mid n} \Phi_{K G}^{d}\left(V^{n / d}\right) .
$$

(The sum on the right-hand side is divisible by $n$ in $R_{K G}$.) The functions $\Phi_{K G}^{n}$ are called the Lie resolvents for $G$ over $K$. As shown in [5],

$$
\Phi_{K G}^{n}(V)=\sum_{d \mid n} \mu(n / d) d L^{d}\left(V^{n / d}\right),
$$

where $\mu$ denotes the Möbius function. Furthermore,

$$
\Phi_{K G}^{n}=\mu(n) \psi_{S}^{n} \quad \text { when } \operatorname{char}(K) \nmid n ;
$$

here $\psi_{S}^{n}$ denotes the $n$th Adams operation on $R_{K G}$ formed by means of symmetric powers (see Section 2 below). In particular, $\Phi_{K G}^{1}$ is the identity function.

Let $G$ be any group and let $K$ be a field of prime characteristic $p$. Define $\mathbb{Z}$ linear functions $\zeta_{K G}^{n}: R_{K G} \rightarrow R_{K G}$ as follows. For $n$ not divisible by $p$ define $\zeta_{K G}^{n}=\mu(n) \psi_{s}^{n}$. In particular, $\zeta_{K G}^{1}$ is the identity function. Define $\zeta_{K G}^{p}=\Phi_{K G}^{p}$, that is, $\zeta_{K G}^{p}(V)=p L^{p}(V)-V^{p}$ for every finite-dimensional $K G$-module $V$. For $k>1$, with $k$ even, define

$$
\zeta_{K G}^{p^{k}}=-p^{k-2}\left(\psi_{S}^{p^{k}}+\zeta_{K G}^{p} \circ \psi_{S}^{p^{k-1}}\right)
$$

(Note that functions are written on the left and o denotes composition of functions.) For $k>1$, with $k$ odd, define

$$
\zeta_{K G}^{p^{k}}=-p^{k-3}\left(\psi_{S}^{p^{k}}+\zeta_{K G}^{p} \circ \psi_{S}^{p^{k-1}}+\zeta_{K G}^{p^{2}} \circ \psi_{S}^{p^{k-2}}\right)
$$

Finally, for $n=p^{k} m$, where $p \nmid m$, define $\zeta_{K G}^{n}=\zeta_{K G}^{p^{k}} \circ \zeta_{K G}^{m}$. Thus the functions $\zeta_{K G}^{n}$ are defined in terms of $p$ th Lie powers and Adams operations. 
THEOREM 1.1. Let $K$ be a field of prime characteristic $p$ and let $G$ be a finite group with a Sylow $p$-subgroup of order at most $p$. Then, for every finite-dimensional $K G$-module $V$,

$$
L^{n}(V)=\frac{1}{n} \sum_{d \mid n} \zeta_{K G}^{d}\left(V^{n / d}\right)
$$

In other words, the Lie resolvents are given by $\Phi_{K G}^{n}=\zeta_{K G}^{n}$ for all $n$. More can be said in the cases where $G$ is a $p^{\prime}$-group and where the Sylow $p$-subgroup is normal: see the beginning of Section 7 and the last part of Section 6, respectively.

COROLlaRY 1.2. Let $K, p, G$ and $V$ be as in the theorem. Let $n$ be a positive integer, and write $n=p^{k} m$ where $p \nmid m$. Then $\Phi_{K G}^{n}=\Phi_{K G}^{p^{k}} \circ \Phi_{K G}^{m}$ and

$$
L^{n}(V)=\frac{1}{p^{k}} \sum_{i=0}^{k} \Phi_{K G}^{p^{i}}\left(L^{m}\left(V^{p^{k-i}}\right)\right) .
$$

The first statement comes from the fact that $\zeta_{K G}^{n}=\zeta_{K G}^{p^{k}} \circ \zeta_{K G}^{m}$, by definition of $\zeta_{K G}^{n}$. The second statement then follows by (1.1): we write each divisor $d$ of $n$ as $d=p^{i} q$, where $0 \leq i \leq k$ and $q \mid m$, and use the facts that $\Phi_{K G}^{d}=\Phi_{K G}^{p^{i}} \circ \Phi_{K G}^{q}$ and each $\Phi_{K G}^{p^{i}}$ is linear. Hence the structure of arbitrary Lie powers is determined by the functions $\Phi_{K G}^{p^{k}}$ and $m$ th Lie powers for integers $m$ not divisible by $p$. It would be interesting to know if the corollary is true for all groups.

If we wish to use Theorem 1.1 for a particular group $G$ we need to be able to calculate the functions $\zeta_{K G}^{n}$. Thus we need to be able to find $\zeta_{K G}^{p}$ (or, equivalently, $p$ th Lie powers) and the Adams operations $\psi_{s}^{n}$. In Sections 6 and 7 we discuss how this might be done provided that enough information is available about the group $G$. The calculation of the $\psi_{s}^{n}$ is simplified a little by the fact that these functions are periodic in $n$, as shown in Section 7. It is clear, however, that there will be significant difficulties in practice except in small special cases such as where the Sylow $p$-subgroup of $G$ is normal and self-centralizing.

\section{Preliminaries}

Throughout this section $K$ is any field. We start by considering an arbitrary group $G$, but in the second half of the section $G$ will be finite.

We have already mentioned the Green ring $R_{K G}$. This is a free $\mathbb{Z}$-module with a basis consisting of the (isomorphism classes of) finite-dimensional indecomposable $K G$-modules. We write $\Gamma_{K G}$ for the Green algebra, defined by $\Gamma_{K G}=\mathbb{C} \otimes_{\mathbb{Z}} R_{K G}$. 
Thus $\Gamma_{K G}$ is a commutative $\mathbb{C}$-algebra. The identity element of $\Gamma_{K G}$, denoted of course by 1 , is the isomorphism class of the trivial one-dimensional $K G$-module.

For any extension field $\widehat{K}$ of $K$ there is a ring homomorphism $\iota: R_{K G} \rightarrow R_{\widehat{K} G}$ determined by $V \mapsto \widehat{K} \otimes_{K} V$ for every finite-dimensional $K G$-module $V$. It follows from the Noether-Deuring Theorem (see [11, (29.7)]) that $\iota$ is an embedding.

If $\theta: A \rightarrow B$ is a homomorphism of groups, then every $K B$-module $V$ can be made into a $K A$-module by taking the action of each element $g$ of $A$ on $V$ to be the same as the action of $\theta(g)$. Thus $\theta$ determines a ring homomorphism $\theta^{*}: R_{K B} \rightarrow R_{K A}$. If $\theta$ is surjective then $\theta^{*}$ is an embedding. If $A$ is a subgroup of $B$ and $\theta$ is the inclusion map then $\theta^{*}$ is called restriction from $B$ to $A$ and, for $V \in R_{K B}$, we sometimes write $V \downarrow_{A}$ instead of $\theta^{*}(V)$.

If $V$ is a finite-dimensional $K G$-module then, for every positive integer $n, L^{n}(V)$ denotes the $n$th Lie power of $V$, as already defined. Similarly, $\wedge^{n}(V)$ denotes the $n$th exterior power of $V$, and $S^{n}(V)$ the $n$th symmetric power of $V$. All of these are finitedimensional $K G$-modules and may be regarded as elements of $R_{K G}$. The exterior and symmetric powers may be encoded by their Hilbert series $\wedge(V, t)$ and $S(V, t)$. These are the power series in an indeterminate $t$ with coefficients in $R_{K G}$ defined by

$$
\begin{aligned}
& \wedge(V, t)=1+\Lambda^{1}(V) t+\Lambda^{2}(V) t^{2}+\cdots, \\
& S(V, t)=1+S^{1}(V) t+S^{2}(V) t^{2}+\cdots .
\end{aligned}
$$

We shall need to use the two types of Adams operations on $R_{K G}$ defined by means of exterior powers and symmetric powers. Following [5] and [6] we denote these by $\psi_{\wedge}^{n}$ and $\psi_{S}^{n}$, respectively. We summarise the basic facts and refer to [5] for further details. In the ring of all symmetric functions in variables $x_{1}, x_{2}, \ldots$, the $n$th power sum may be written as a polynomial in the elementary symmetric functions and as a polynomial in the complete symmetric functions:

$$
x_{1}^{n}+x_{2}^{n}+\cdots=\rho_{n}\left(e_{1}, \ldots, e_{n}\right)=\sigma_{n}\left(h_{1}, \ldots, h_{n}\right) \text {. }
$$

For each positive integer $n, \psi_{\wedge}^{n}$ and $\psi_{S}^{n}$ are $\mathbb{Z}$-linear functions on $R_{K G}$ such that, for every finite-dimensional $K G$-module $V$,

$$
\begin{gathered}
\psi_{\wedge}^{n}(V)=\rho_{n}\left(\Lambda^{1}(V), \ldots, \wedge^{n}(V)\right), \quad \psi_{S}^{n}(V)=\sigma_{n}\left(S^{1}(V), \ldots, S^{n}(V)\right), \\
\psi_{\wedge}^{1}(V)-\psi_{\wedge}^{2}(V) t+\psi_{\wedge}^{3}(V) t^{2}-\cdots=\frac{d}{d t} \log \wedge(V, t), \\
\psi_{S}^{1}(V)+\psi_{S}^{2}(V) t+\psi_{S}^{3}(V) t^{2}+\cdots=\frac{d}{d t} \log S(V, t) .
\end{gathered}
$$

Also, $\psi_{\wedge}^{n}=\psi_{s}^{n}$ when $\operatorname{char}(K) \nmid n$. Furthermore, the following result was established in [5, Theorem 5.4]. 
LEMMA 2.1. Let $q$ and $n$ be positive integers such that $q$ is not divisible by char $(K)$. Then $\psi_{\wedge}^{q} \circ \psi_{\wedge}^{n}=\psi_{\wedge}^{q n}$ and $\psi_{S}^{q} \circ \psi_{S}^{n}=\psi_{S}^{q n}$.

In Section 1 we described the basic properties of the Lie resolvents $\Phi_{K G}^{n}$. Like the Adams operations, these are $\mathbb{Z}$-linear functions on $R_{K G}$. Also, in Section 1 , we defined $\mathbb{Z}$-linear functions $\zeta_{K G}^{n}$ on $R_{K G}$ in the case where $K$ has prime characteristic $p$. We shall establish some elementary properties of these various functions on $R_{K G}$. Whenever we discuss $\zeta_{K G}^{n}$ we assume implicitly that $K$ has prime characteristic $p$.

LEMMA 2.2. Let $\theta: A \rightarrow B$ be a homomorphism of groups, yielding the ring homomorphism $\theta^{*}: R_{K B} \rightarrow R_{K A}$. Then, for every positive integer $n$ and every finite-dimensional $K B$-module $V$,

$$
L^{n}\left(\theta^{*}(V)\right)=\theta^{*}\left(L^{n}(V)\right), \quad \bigwedge^{n}\left(\theta^{*}(V)\right)=\theta^{*}\left(\bigwedge^{n}(V)\right), \quad S^{n}\left(\theta^{*}(V)\right)=\theta^{*}\left(S^{n}(V)\right)
$$

PROOF. This is straightforward.

LEMMA 2.3. Let $\theta: A \rightarrow B$ be a homomorphism of groups, yielding the ring homomorphism $\theta^{*}: R_{K B} \rightarrow R_{K A}$. Then, for every positive integer $n$,

$$
\begin{aligned}
\psi_{\wedge}^{n} \circ \theta^{*} & =\theta^{*} \circ \psi_{\wedge}^{n}, & \psi_{S}^{n} \circ \theta^{*} & =\theta^{*} \circ \psi_{S}^{n}, \\
\Phi_{K A}^{n} \circ \theta^{*} & =\theta^{*} \circ \Phi_{K B}^{n}, & \zeta_{K A}^{n} \circ \theta^{*} & =\theta^{*} \circ \zeta_{K B}^{n} .
\end{aligned}
$$

PROOF. The results for $\psi_{\wedge}^{n}, \psi_{S}^{n}$ and $\Phi_{K G}^{n}$ follow from (2.2), (1.2) and Lemma 2.2. The result for $\zeta_{K G}^{n}$ follows from its definition.

LEMMA 2.4. Let $\iota: R_{K G} \rightarrow R_{\widehat{K} G}$ be the ring embedding associated with an extension field $\widehat{K}$ of $K$. Then, for every positive integer $n$ and every finite-dimensional KG-module $V$,

$$
\begin{gathered}
L^{n}(\iota(V))=\iota\left(L^{n}(V)\right), \quad \wedge^{n}(\iota(V))=\iota\left(\bigwedge^{n}(V)\right), \quad S^{n}(\iota(V))=\imath\left(S^{n}(V)\right), \\
\psi_{\wedge}^{n} \circ \iota=\iota \circ \psi_{\wedge}^{n}, \quad \psi_{S}^{n} \circ \iota=\imath \circ \psi_{S}^{n}, \quad \Phi_{\widehat{K} G}^{n} \circ \iota=\imath \circ \Phi_{K G}^{n}, \quad \zeta_{\widehat{K} G}^{n} \circ \iota=\iota \circ \zeta_{K G}^{n} .
\end{gathered}
$$

PROOF. This is similar to the proof of Lemmas 2.2 and 2.3.

LEMMA 2.5. Let $V$ be a finite-dimensional $K G$-module, and I a one-dimensional $K G$-module. Then, for every positive integer $n$,

$$
\begin{aligned}
L^{n}(I V) & =I^{n} L^{n}(V), & \bigwedge^{n}(I V) & =I^{n} \wedge^{n}(V), & S^{n}(I V) & =I^{n} S^{n}(V), \\
\psi_{\wedge}^{n}(I V) & =I^{n} \psi_{\wedge}^{n}(V), & \psi_{S}^{n}(I V) & =I^{n} \psi_{S}^{n}(V), & \Phi_{K G}^{n}(I V) & =I^{n} \Phi_{K G}^{n}(V), \\
\zeta_{K G}^{n}(I V) & =I^{n} \zeta_{K G}^{n}(V), & \psi_{\wedge}^{n}(I) & =\psi_{S}^{n}(I)=I^{n}, & \Phi_{K G}^{n}(I) & =\zeta_{K G}^{n}(I)=\mu(n) I^{n} .
\end{aligned}
$$


PROOF. This is mostly straightforward. For the statement about $\Phi_{K G}^{n}(I)$, note that $L^{d}\left(I^{n / d}\right)=0$ for divisors $d$ of $n$ such that $d>1$. The statement about $\zeta_{K G}^{n}(I)$ comes easily from its definition, using the results for $\psi_{S}^{n}(I)$ and $\Phi_{K G}^{p}(I)$.

From now on in this section, assume that $G$ is finite, and write $p=\operatorname{char}(K)$. (We are particularly interested in the case where $p \neq 0$.) Let $\widehat{K}$ be the algebraic closure of $K$ and let $G_{p}$, be the set of all elements of $G$ of order not divisible by $p$. Let $\Delta$ be the $\mathbb{C}$-algebra consisting of all class functions from $G_{p^{\prime}}$ to $\mathbb{C}$, that is, functions $\delta$ such that $\delta(g)=\delta\left(g^{\prime}\right)$ whenever $g$ and $g^{\prime}$ are elements of $G_{p^{\prime}}$ which are conjugate in $G$. Let $c$ be the least common multiple of the orders of the elements of $G_{p^{\prime}}$, and choose and fix primitive $c$ th roots of unity $\xi$ in $\widehat{K}$ and $\omega$ in $\mathbb{C}$. Then, for every finite-dimensional $K G$-module $V$ we may define the Brauer character of $V$ to be the element $\operatorname{Br}(V)$ of $\Delta$ such that if $g \in G_{p^{\prime}}$ has eigenvalues $\xi^{k_{1}}, \ldots, \xi^{k_{r}}$ in its action on $V$ then $\operatorname{Br}(V)(g)=\omega^{k_{1}}+\cdots+\omega^{k_{r}}$. (See [3, Section 5.3].) Furthermore, we may extend the definition linearly so that $\operatorname{Br}(V)$ is defined for an arbitrary element $V$ of $\Gamma_{K G}$. Then $\mathrm{Br}: \Gamma_{K G} \rightarrow \Delta$ is a $\mathbb{C}$-algebra homomorphism.

For each positive integer $n$, define a function $\psi_{0}^{n}: \Delta \rightarrow \Delta$ by $\psi_{0}^{n}(\delta)(g)=\delta\left(g^{n}\right)$ for all $\delta \in \Delta$ and $g \in G_{p^{\prime}}$. Clearly $\psi_{0}^{n}$ is an algebra endomorphism of $\Delta$ and

$$
\psi_{0}^{m} \circ \psi_{0}^{n}=\psi_{0}^{m n},
$$

for all positive integers $m$ and $n$.

LEMMA 2.6. Let $V$ be a finite-dimensional $K G$-module. Then, for all $n$,

$$
\operatorname{Br}\left(\psi_{\wedge}^{n}(V)\right)=\psi_{0}^{n}(\operatorname{Br}(V))=\operatorname{Br}\left(\psi_{S}^{n}(V)\right) .
$$

PROOF. This is well known: however, for the reader's convenience we sketch a proof. If $g \in G_{p^{\prime}}$ has eigenvalues $\xi^{k_{1}}, \ldots, \xi^{k_{r}}$ on $V$, then, for $i=1, \ldots, n$,

$$
\operatorname{Br}\left(\bigwedge^{i}(V)\right)(g)=e_{i}\left(\omega^{k_{1}}, \ldots, \omega^{k_{r}}\right), \quad \operatorname{Br}\left(S^{i}(V)\right)(g)=h_{i}\left(\omega^{k_{1}}, \ldots, \omega^{k_{r}}\right) .
$$

Thus, by (2.2) and (2.1),

$$
\begin{aligned}
\operatorname{Br}\left(\psi_{\wedge}^{n}(V)\right)(g) & =\rho_{n}\left(e_{1}\left(\omega^{k_{1}}, \ldots, \omega^{k_{r}}\right), \ldots, e_{n}\left(\omega^{k_{1}}, \ldots, \omega^{k_{r}}\right)\right) \\
& =\omega^{k_{1} n}+\cdots+\omega^{k_{n} n}=\operatorname{Br}(V)\left(g^{n}\right)=\psi_{0}^{n}(\operatorname{Br}(V))(g) .
\end{aligned}
$$

This gives the result for $\psi_{\wedge}^{n}$. The result for $\psi_{S}^{n}$ is similar.

The following result is Brandt's character formula [4], as generalised to Brauer characters (see, for example, [7, (5.4)] or [17, (2.11)]). 
LEMMA 2.7. Let $V$ be a finite-dimensional $K G$-module. Then, for all $n$,

$$
\operatorname{Br}\left(L^{n}(V)\right)=\frac{1}{n} \sum_{d \mid n} \mu(d) \psi_{0}^{d}\left(\operatorname{Br}\left(V^{n / d}\right)\right) .
$$

We can now calculate the Brauer characters associated with $\Phi_{K G}^{n}$ and $\zeta_{K G}^{n}$.

LEMMA 2.8. Let $V$ be a finite-dimensional $K G-\operatorname{module.~Then,~for~all~} n$,

$$
\operatorname{Br}\left(\Phi_{K G}^{n}(V)\right)=\mu(n) \psi_{0}^{n}(\operatorname{Br}(V))=\operatorname{Br}\left(\zeta_{K G}^{n}(V)\right) .
$$

PROOF. By (1.1), $\operatorname{Br}\left(L^{n}(V)\right)=\frac{1}{n} \sum_{d \mid n} \operatorname{Br}\left(\Phi_{K G}^{d}\left(V^{n / d}\right)\right)$. Hence, by Lemma 2.7 and induction on $n$, we have $\operatorname{Br}\left(\Phi_{K G}^{n}(V)\right)=\mu(n) \psi_{0}^{n}(\operatorname{Br}(V))$. It remains to prove that $\operatorname{Br}\left(\zeta_{K G}^{n}(V)\right)=\mu(n) \psi_{0}^{n}(\operatorname{Br}(V))$ for all $n$.

If $p \nmid n$ then $\zeta_{K G}^{n}(V)=\mu(n) \psi_{S}^{n}(V)$ and the result follows by Lemma 2.6. Also, $\zeta_{K G}^{p}=\Phi_{K G}^{p}$, so the result for $\zeta_{K G}^{p}$ follows from the first part. This implies that $\operatorname{Br}\left(\zeta_{K G}^{p}(U)\right)=-\psi_{0}^{p}(\operatorname{Br}(U))$ for all $U \in R_{K G}$.

Suppose that $k>1$ and $k$ is even. Then, by the definition of $\zeta_{K G}^{p^{k}}$,

$$
\operatorname{Br}\left(\zeta_{K G}^{p^{k}}(V)\right)=-p^{k-2} \operatorname{Br}\left(\psi_{S}^{p^{k}}(V)\right)-p^{k-2} \operatorname{Br}\left(\zeta_{K G}^{p}\left(\psi_{S}^{p^{k-1}}(V)\right)\right) .
$$

Hence, by Lemma 2.6 and the result for $\zeta_{K G}^{p}$,

$$
\operatorname{Br}\left(\zeta_{K G}^{p^{k}}(V)\right)=-p^{k-2} \psi_{0}^{p^{k}}(\operatorname{Br}(V))+p^{k-2} \psi_{0}^{p}\left(\psi_{0}^{p^{k-1}}(\operatorname{Br}(V))\right) .
$$

Therefore, by (2.5), $\operatorname{Br}\left(\zeta_{K G}^{p^{k}}(V)\right)=0=\mu\left(p^{k}\right) \psi_{0}^{p^{k}}(\operatorname{Br}(V))$. Thus the result holds for $\zeta_{K G}^{p^{k}}$. The result for $\zeta_{K G}^{p^{k}}$ when $k>1$ and $k$ is odd is proved in a similar way using the results for $\zeta_{K G}^{p}$ and $\zeta_{K G}^{p^{2}}$.

Now suppose that $n=p^{k} m$, where $p \nmid m$. Then, by the definition of $\zeta_{K G}^{n}$,

$$
\begin{aligned}
\operatorname{Br}\left(\zeta_{K G}^{n}(V)\right) & =\operatorname{Br}\left(\zeta_{K G}^{p^{k}}\left(\zeta_{K G}^{m}(V)\right)\right)=\mu\left(p^{k}\right) \psi_{0}^{p^{k}}\left(\operatorname{Br}\left(\zeta_{K G}^{m}(V)\right)\right) \\
& =\mu\left(p^{k}\right) \psi_{0}^{p^{k}}\left(\mu(m) \psi_{0}^{m}(\operatorname{Br}(V))\right)=\mu(n) \psi_{0}^{n}(\operatorname{Br}(V)) .
\end{aligned}
$$

This is the required result.

Recall that $R_{K G}$ has a $\mathbb{Z}$-basis consisting of the finite-dimensional indecomposable $K G$-modules. Let $\left(R_{K G}\right)_{\text {proj }}$ and $\left(R_{K G}\right)_{\text {nonp }}$ be the $\mathbb{Z}$-submodules spanned, respectively, by the projective and the non-projective indecomposables. Then, for $V \in R_{K G}$, we can write $V=V_{\text {proj }}+V_{\text {nonp }}$, uniquely, where $V_{\text {proj }} \in\left(R_{K G}\right)_{\text {proj }}$ and $V_{\text {nonp }} \in\left(R_{K G}\right)_{\text {nonp. }}$.

LEMMA 2.9. Let $U, V \in R_{K G}$. If $U_{\text {nonp }}=V_{\text {nonp }}$ and $\operatorname{Br}(U)=\operatorname{Br}(V)$ then $U=V$. In particular, if $G$ is a $p^{\prime}$-group and $\operatorname{Br}(U)=\operatorname{Br}(V)$ then $U=V$.

PROOF. The hypotheses yield $\operatorname{Br}\left(U_{\text {proj }}\right)=\operatorname{Br}\left(V_{\text {proj }}\right)$. However, if $W$ and $W^{\prime}$ are finite-dimensional projective $K G$-modules such that $\operatorname{Br}(W)=\operatorname{Br}\left(W^{\prime}\right)$ then $W \cong W^{\prime}$ (see [3, Corollary 5.3.6]). Thus $U_{\text {proj }}=V_{\text {proj }}$, and so $U=V$. 


\section{Exterior and symmetric powers}

Throughout this section, let $K$ be a field of prime characteristic $p$ and let $G$ be a finite group with a normal Sylow $p$-subgroup of order $p$. As we shall see, there are certain basic indecomposable $K G$-modules $J_{1}, J_{2}, \ldots, J_{p}$. The main purpose of this section is to give formulae for the power series $\Lambda\left(J_{r}, t\right)$ and $S\left(J_{r}, t\right)$. The formula for $\wedge\left(J_{r}, t\right)$ is due to Kouwenhoven [15] and was also proved by Hughes and Kemper [14]. The formula for $S\left(J_{r}, t\right)$ is a corollary of a result in [14].

Kouwenhoven's results are primarily concerned with $\mathrm{GL}(2, p)$ and go beyond what is required here. In order to keep the treatment as simple as possible we have therefore chosen to follow [14]. However, we use slightly different notation and we consider right $K G$-modules instead of left $K G$-modules. If $V$ is a left $K G$-module then $V$ becomes a right $K G$-module by defining $v g=g^{-1} v$ for all $v \in V, g \in G$. This gives a one-one correspondence between left and right $K G$-modules. We shall use this correspondence in order to interpret the results of [14] as results about right $K G$ modules, noting that the correspondence commutes with taking direct sums, tensor products, exterior powers and symmetric powers.

Let $P$ be the (normal) Sylow $p$-subgroup of $G$. Thus $P$ has a complement in $G$, and $G$ is a semidirect product, $G=H P$, where $H$ is a $p^{\prime}$-group. Let $P=\left\{1, a, \ldots, a^{P-1}\right\}$. There is a right action of $P$ on the group algebra $K P$ given by multiplication and a right action of $H$ given by $a^{i} \mapsto h^{-1} a^{i} h$ for all $h \in H$ and $i=0, \ldots, p-1$. In this way $K P$ becomes a right $K G$-module. For $r=1, \ldots, p$, the $r$ th power of the augmentation ideal is $K P(a-1)^{r}$, and this is invariant under the action of $G$. Thus, for $r=1, \ldots, p$, we obtain a right $K G$-module $J_{r}$ defined by $J_{r}=K P / K P(a-1)^{r}$. It is easily verified that $J_{r}$ has dimension $r$ and corresponds to the left module $V_{r}$ of [14]. (Also, the isomorphism class of $J_{r}$ does not depend on the choice of complement $H$.) Furthermore, $J_{1}=1$ in the Green ring $R_{K G}$.

For each $h \in H$, let $m(h)$ be the element of $\{1, \ldots, p-1\}$ determined by $h^{-1} a h=a^{m(h)}$, and let $m(h)$ also denote the corresponding element of the prime subfield of $K$. There is then a homomorphism $\alpha: H \rightarrow K \backslash\{0\}$ given by $\alpha(h)=m(h)$ for all $h$. This yields a one-dimensional right $K H$-module, which we also denote by $\alpha$. Furthermore, we regard $\alpha$ as a right $K G$-module, by means of the projection $G \rightarrow H$. It is easily verified that this module corresponds to the left $K G$-module denoted by $V_{\alpha}$ or $\alpha$ in [14]. In $R_{K G}$, as in $R_{K H}$, we have $\alpha^{p-1}=1$. Indeed, $\alpha$ has multiplicative order $q$ where $q=\left|H / C_{H}(P)\right|$.

As shown by the pullback construction described in [14], there exists a finite $p^{\prime}$ group $\widetilde{H}$ and an extension field $\widehat{K}$ of $K$ with homomorphisms $\theta: \widetilde{H} \rightarrow H$ and $\beta: \widetilde{H} \rightarrow \widehat{K} \backslash\{0\}$ such that $\theta$ is surjective and $\beta(h)^{2}=\alpha(\theta(h))$ for all $h \in \widetilde{H}$. Let $\widetilde{G}$ be the semidirect product $\widetilde{H} P$ with $P$ normal such that, for all $h \in \widetilde{H}$, the action of $h$ on $P$ by conjugation is given by the action of $\theta(h)$. Thus $\theta$ extends to a surjective 
homomorphism $\theta: \widetilde{G} \rightarrow G$ which is the identity on $P$.

We regard the ring $R_{K G}$ as a subring of $R_{\widehat{K} G}$ by means of the embedding $\iota: R_{K G} \rightarrow$ $R_{\widehat{K} G}$ described at the beginning of Section 2 . Also, we regard $R_{\widehat{K} G}$ as a subring of $R_{\widehat{K} \tilde{G}}$ by means of the embedding $\theta^{*}$ obtained from $\theta: \widetilde{G} \rightarrow G$, as described in Section 2 . Thus $R_{K G}$ is a subring of $R_{\widetilde{K}} \tilde{G}$. It is easily verified that the images under $\theta^{*} \circ \iota$ of the $K G$-modules $J_{r}$ and $\alpha$ are isomorphic to the $\widehat{K} \widetilde{G}$-modules defined in the same way for $\widetilde{G}$ over $\widehat{K}$. Thus there is no conflict of notation. By Lemmas 2.2 and 2.4, the exterior and symmetric powers of $J_{r}$ in $R_{K G}$ are the same as the exterior and symmetric powers of $J_{r}$ in $R_{\widehat{K}} \tilde{G}$. Thus we may use $R_{\widehat{K}} \tilde{G}$ in order to find expressions for $\wedge\left(J_{r}, t\right)$ and $S\left(J_{r}, t\right)$.

We regard $R_{\widehat{K} \widetilde{H}}$ as a subring of $R_{\widehat{K}} \tilde{G}$ by means of the embedding given by the projection $\widetilde{G} \rightarrow \widetilde{H}$. Clearly $\alpha \in R_{\widetilde{K} \tilde{H}}$. The homomorphism $\beta: \widetilde{H} \rightarrow \widehat{K} \backslash\{0\}$ yields an element of $R_{\widetilde{K} \tilde{H}}$ which we also denote by $\beta$. From the properties of $\beta$ we see that $\beta^{2}=\alpha$. Hence $\beta^{2 p-2}=1$ and $\beta^{-1}$ exists. Note that if $p=2$ we have $\alpha=1$ and $\operatorname{char}(\widehat{K})=2$ : thus the definition of $\beta$ gives $\beta=1$ in this case.

As in [14], but using $\lambda$ instead of $\mu$ to avoid the notation for the Möbius function, we extend $R_{\widehat{K}} \widetilde{G}$ by an element $\lambda$ satisfying $\lambda^{2}-\beta^{-1} J_{2} \lambda+1=0$ to form a commutative ring $R_{\widetilde{K} \widetilde{G}}[\lambda]$. Note that this is a free $R_{\widetilde{K} \widetilde{G}}$-module: $R_{\widetilde{K} \widetilde{G}}[\lambda]=R_{\widetilde{K} \widetilde{G}} \oplus R_{\widetilde{K} \widetilde{G}} \lambda$. Also, $\lambda$ is invertible in $R_{\hat{K} \widetilde{G}}[\lambda]$. We shall find expressions for $\wedge\left(J_{r}, t\right)$ and $S\left(J_{r}, t\right)$ as elements of the power series ring $R_{\widehat{K} \widetilde{G}}[\lambda][[t]]$.

By [14, Lemma 1.3],

$$
J_{r}=\beta^{r-1} \sum_{j=0}^{r-1} \lambda^{r-1-2 j},
$$

for $r=1, \ldots, p$. Also, by [14, Theorem 1.4], $R_{\widehat{K} \widetilde{G}}[\lambda]$ is generated by $R_{\widehat{K} \widetilde{H}}$ and $\lambda$, that is, $R_{\widehat{K} \widetilde{G}}[\lambda]=R_{\widehat{K} \tilde{H}}[\lambda]$. Tensoring with $\mathbb{C}$ we obtain $\Gamma_{\widehat{K} \tilde{G}}[\lambda]=\Gamma_{\widehat{K} \tilde{H}}[\lambda]$, where $\Gamma_{\widehat{K} \tilde{G}}=\mathbb{C} \otimes R_{\widehat{K}} \tilde{G}$ and $\Gamma_{\widehat{K} \tilde{H}}=\mathbb{C} \otimes R_{\widehat{K} \tilde{H}}$.

By [12, (81.90)], the algebra $\Gamma_{\widehat{K} \widetilde{G}}$ is semisimple. Thus it is isomorphic to the direct sum of $m$ copies of $\mathbb{C}$, where $m$ is the number of indecomposable $\widehat{K} \widetilde{G}$-modules. Thus there are exactly $m$ non-zero algebra homomorphisms $\Gamma_{\widehat{K}} \widetilde{G} \rightarrow \mathbb{C}$. The restrictions to $R_{\widehat{K} \tilde{G}}$ of these homomorphisms are called the 'species' of $R_{\widehat{K} \widetilde{G}}$. Note that if $U, V \in R_{\widehat{K} \widetilde{G}}$ and $\phi(U)=\phi(V)$ for every species $\phi$ then $U=V$.

Let $M_{2 p}^{*}$ denote the subset of $\mathbb{C}$ consisting of all $2 p$ th roots of unity except for 1 and -1 . Thus $\gamma^{2 p-2}+\gamma^{2 p-4}+\cdots+\gamma^{2}+1=0$ for all $\gamma \in M_{2 p}^{*}$. By the proof of [14, Theorem 1.6], for each $\gamma \in\left\{\beta, \beta^{-1}\right\} \cup M_{2 p}^{*}$ there is a $\mathbb{C}$-algebra homomorphism $\phi_{Y}: \Gamma_{\widehat{K} \tilde{G}}[\lambda] \rightarrow \Gamma_{\widehat{K} \tilde{H}}$ given by $\phi_{Y}(\chi)=\chi$ for all $\chi \in \Gamma_{\widehat{K} \tilde{H}}$ and $\phi_{\gamma}(\lambda)=\gamma$. Also, for each $h \in \widetilde{H}$ there is a $\mathbb{C}$-algebra homomorphism $\varepsilon_{h}: \Gamma_{\widehat{K} \widetilde{H}} \rightarrow \mathbb{C}$ such that, for all $\chi \in \Gamma_{\widehat{K} \tilde{H}}, \varepsilon_{h}(\chi)$ is the value at $h$ of the Brauer character of $\chi$, that is, $\varepsilon_{h}(\chi)=\operatorname{Br}(\chi)(h)$. For $\gamma \in\left\{\beta, \beta^{-1}\right\} \cup M_{2 p}^{*}$ and $h \in \tilde{H}$, let $\phi_{h, \gamma}=\varepsilon_{h} \circ \phi_{\gamma}$. Thus 
$\phi_{h, y}$ is a $\mathbb{C}$-algebra homomorphism $\phi_{h, y}: \Gamma_{\widehat{K}} \tilde{G}[\lambda] \rightarrow \mathbb{C}$. The following result is [14, Theorem 1.6], apart from minor notational differences.

LEMMA 3.1. For each $\gamma \in\left\{\beta, \beta^{-1}\right\} \cup M_{2 p}^{*}$ and each $h \in \widetilde{H}$, the restriction of $\phi_{h, \gamma}$ to $R_{\widehat{K}} \tilde{G}$ is a species of $R_{\widetilde{K}} \tilde{G}$. The homomorphisms $\phi_{h, \gamma}$ and $\phi_{h^{\prime}, \gamma^{\prime}}$ restrict to the same species if and only if $h$ and $h^{\prime}$ are conjugate in $\widetilde{H}$ and $\gamma^{\prime} \in\left\{\gamma, \gamma^{-1}\right\}$. Every species of $R_{\widehat{K} \tilde{G}}$ arises as the restriction of some $\phi_{h, \gamma}$.

In particular, $\phi_{h, \beta}$ gives the same species as $\phi_{h, \beta^{-1}}$. Since elements of $R_{\widetilde{K} \widetilde{G}}$ are determined by their images under the species, we obtain the following result.

COROLLARY 3.2. Let $U, V \in R_{\widehat{K}} \tilde{G}$. If $\phi_{h, \gamma}(U)=\phi_{h, \gamma}(V)$ for all $\gamma \in\{\beta\} \cup M_{2 p}^{*}$ and all $h \in \widetilde{H}$, or if $\phi_{\gamma}(U)=\phi_{\gamma}(V)$ for all $\gamma \in\{\beta\} \cup M_{2 p}^{*}$, then $U=V$.

The description of $\wedge\left(J_{r}, t\right)$ is as follows.

THEOREM 3.3 ([15, Lemma, page 1709]; [14, Theorem 1.10]). For $r=1, \ldots, p$,

$$
\wedge\left(J_{r}, t\right)=\prod_{j=0}^{r-1}\left(1+\beta^{r-1} \lambda^{r-1-2 j} t\right) .
$$

We write $W=J_{p}-\alpha J_{p-1}$ and $\bar{\alpha}=1+\alpha+\cdots+\alpha^{p-2}$, recalling that $\alpha^{p-1}=1$. By direct calculation from (3.1) we get the following result.

LEMMA 3.4. For the homomorphisms $\phi_{\beta}$ and $\phi_{\gamma}$, where $\gamma \in M_{2 p}^{*}$, we have

$$
\begin{array}{lll}
\phi_{\beta}\left(J_{p}\right)=1+\bar{\alpha}, & \phi_{\beta}\left(J_{p-1}\right)=\bar{\alpha}, & \phi_{\beta}(W)=1, \\
\phi_{\gamma}\left(J_{p}\right)=0, & \phi_{\gamma}\left(J_{p-1}\right)=-\gamma^{p} \beta^{p-2}, & \phi_{\gamma}(W)=\gamma^{p} \beta^{p} .
\end{array}
$$

For $r=1, \ldots, p$, write

$$
X_{r}=\left(1-W^{r-1} t^{p}\right)\left(1-t^{p}\right)^{-1}\left(1-\Lambda^{1}\left(J_{r}\right) t+\Lambda^{2}\left(J_{r}\right) t^{2}-\cdots\right)^{-1} .
$$

Thus, by Theorem 3.3,

$$
X_{r}=\left(1-W^{r-1} t^{p}\right)\left(1-t^{p}\right)^{-1} \prod_{j=0}^{r-1}\left(1-\beta^{r-1} \lambda^{r-1-2 j} t\right)^{-1} .
$$

Let the homomorphisms $\phi_{\beta}$ and $\phi_{\gamma}$ act on $\Gamma_{\widehat{K}} \tilde{G}[\lambda][[t]]$ by action on coefficients. Then it is easily verified that $\phi_{\beta}\left(X_{r}\right)=\prod_{j=0}^{r-1}\left(1-\alpha^{j} t\right)^{-1}$ and, for $\gamma \in M_{2 p}^{*}$,

$$
\phi_{\gamma}\left(X_{r}\right)=\left(1-\beta^{p(r-1)} \gamma^{p(r-1)} t^{p}\right)\left(1-t^{p}\right)^{-1} \prod_{j=0}^{r-1}\left(1-\beta^{r-1} \gamma^{r-1-2 j} t\right)^{-1} .
$$


Replacing $\alpha$ by $\operatorname{Br}(\alpha)(h)$ and $\beta$ by $\operatorname{Br}(\beta)(h)$, for $h \in \widetilde{H}$, we obtain expressions for $\phi_{h, \beta}\left(X_{r}\right)$ and $\phi_{h, \gamma}\left(X_{r}\right)$. Comparison with [14, Proposition 1.13] shows that $\phi_{h, \beta}\left(X_{r}\right)=$ $\phi_{h, \beta}\left(S\left(J_{r}, t\right)\right)$ and $\phi_{h, \gamma}\left(X_{r}\right)=\phi_{h, \gamma}\left(S\left(J_{r}, t\right)\right)$. Therefore, by Corollary 3.2, $X_{r}=$ $S\left(J_{r}, t\right)$. Thus we have the following result.

THEOREM 3.5 (based on [14, Proposition 1.13]). For $r=1, \ldots, p$,

$$
\begin{aligned}
S\left(J_{r}, t\right) & =\left(1-\left(J_{p}-\alpha J_{p-1}\right)^{r-1} t^{p}\right)\left(1-t^{p}\right)^{-1} \bigwedge\left(J_{r},-t\right)^{-1} \\
& =\left(1-\left(J_{p}-\alpha J_{p-1}\right)^{r-1} t^{p}\right)\left(1-t^{p}\right)^{-1} \prod_{j=0}^{r-1}\left(1-\beta^{r-1} \lambda^{r-1-2 j} t\right)^{-1} .
\end{aligned}
$$

\section{Adams operations}

We continue to use all the notation of Section 3. In particular, $G$ is a finite group with a normal Sylow $p$-subgroup of order $p$. We shall find expressions for the elements $\psi_{\wedge}^{n}\left(J_{r}\right)$ and $\psi_{S}^{n}\left(J_{r}\right)$ of $R_{K G}$. By Lemmas 2.3 and 2.4, it suffices to find such expressions within $R_{\widehat{K} \widetilde{G}}$. Recall that $\alpha^{p-1}=1$ and $\beta^{2}=\alpha$, so that $\beta^{2 p-2}=1$. For $r \in\{1, \ldots, p\}$, we write $\alpha_{r}=1+\alpha+\cdots+\alpha^{r-1}$. Of particular importance is $\alpha_{p-1}$, which we also denote by $\bar{\alpha}$, as in Lemma 3.4 above. For each non-negative integer $i$, we have $\alpha^{i} \bar{\alpha}=\bar{\alpha}$. Thus $\alpha_{r} \bar{\alpha}=r \bar{\alpha}$. The identity element of $R_{\widehat{K}} \tilde{G}[\lambda]$ is denoted by 1 or $J_{1}$, as convenient. As in Section 3, let $W=J_{p}-\alpha J_{p-1}$.

LEMMA 4.1. For every non-negative integer $n$,

$$
W^{n}= \begin{cases}-\beta^{n+1} J_{p-1}+J_{p} & \text { if } n \text { is odd } \\ \beta^{n} J_{1}+\left(1-\beta^{n}\right) J_{p} & \text { if } n \text { is even. }\end{cases}
$$

PROOF. We use the homomorphisms $\phi_{\beta}$ and $\phi_{\gamma}$, for $\gamma \in M_{2 p}^{*}$, as defined in Section 3. Note that these homomorphisms fix $\alpha$ and $\beta$. Suppose that $n$ is odd. Then, by Lemma 3.4, we find $\phi_{\beta}\left(W^{n}\right)=1=\phi_{\beta}\left(-\beta^{n+1} J_{p \sim 1}+J_{p}\right)$ and

$$
\phi_{\gamma}\left(W^{n}\right)=\gamma^{p} \beta^{n+p-1}=\phi_{\gamma}\left(-\beta^{n+1} J_{p-1}+J_{p}\right) .
$$

Thus, by Corollary 3.2, $W^{n}=-\beta^{n+1} J_{p-1}+J_{p}$. The proof for even $n$ is similar.

By Theorem 3.3 and (2.3),

$$
\psi_{\wedge}^{1}\left(J_{r}\right)-\psi_{\wedge}^{2}\left(J_{r}\right) t+\psi_{\wedge}^{3}\left(J_{r}\right) t^{2}-\cdots=\sum_{j=0}^{r-1} \beta^{r-1} \lambda^{r-1-2 j}\left(1+\beta^{r-1} \lambda^{r-1-2 j} t\right)^{-1}
$$


Hence, as stated in [15, page 1720],

$$
\psi_{\wedge}^{n}\left(J_{r}\right)=\beta^{(r-1) n} \sum_{j=0}^{r-1} \lambda^{(r-1-2 j) n} \text { for all } r \text { and } n .
$$

THEOREM 4.2. Let $k$ be a positive integer and let $r \in\{1, \ldots, p\}$. If $r$ is odd,

$$
\psi_{\wedge}^{p^{k}}\left(J_{r}\right)=r \beta^{r-1}\left(J_{1}-J_{p}\right)+\alpha_{r} J_{p} .
$$

If $p=2$,

If $p$ is odd and $r$ is even,

$$
\psi_{\wedge}^{2^{k}}\left(J_{2}\right)= \begin{cases}2\left(J_{2}-J_{1}\right) & \text { if } k=1 \\ 2 J_{1} & \text { if } k \geq 2\end{cases}
$$

$$
\psi_{\wedge}^{p^{k}}\left(J_{r}\right)= \begin{cases}-r \beta^{r} J_{p-1}+\alpha_{r} J_{p} & \text { if } k \text { is odd } \\ -r \beta^{r+p-1} J_{p-1}+\alpha_{r} J_{p} & \text { if } k \text { is even. }\end{cases}
$$

Proof. We assume that $p$ is odd, noting that the proof for $p=2$ is similar but much easier. Suppose first that $r$ is odd. By (4.1),

$$
\phi_{\beta}\left(\psi_{\wedge}^{p^{k}}\left(J_{r}\right)\right)=\beta^{(r-1) p^{k}} \sum_{j=0}^{r-k} \beta^{(r-1-2 j) p^{k}}=\sum_{j=0}^{r-1} \alpha^{(r-1-j) p^{k}}=\sum_{j=0}^{r-1} \alpha^{r-1-j}=\alpha_{r} .
$$

Also, by Lemma 3.4,

$$
\phi_{\beta}\left(r \beta^{r-1}\left(J_{1}-J_{p}\right)+\alpha_{r} J_{p}\right)=-r \beta^{r-1} \bar{\alpha}+\alpha_{r}(1+\bar{\alpha})=-r \bar{\alpha}+\alpha_{r}+r \bar{\alpha}=\alpha_{r} .
$$

For $\gamma \in M_{2 p}^{*},(4.1)$ gives

$$
\phi_{\gamma}\left(\psi_{\wedge}^{p^{k}}\left(J_{r}\right)\right)=\beta^{(r-1) p^{k}} \sum_{j=0}^{r-1} \gamma^{(r-1-2 j) p^{k}}=r \beta^{(r-1) p^{k}}=r \beta^{r-1} .
$$

Also, by Lemma 3.4, $\phi_{\gamma}\left(r \beta^{r-1}\left(J_{1}-J_{p}\right)+\alpha_{r} J_{p}\right)=r \beta^{r-1}$. Thus, for $r$ odd, the result follows by Corollary 3.2.

Now suppose that $r$ is even. Note that $r+p-p^{k} \equiv r(\bmod 2 p-2)$ if $k$ is odd, and $r+p-p^{k} \equiv r+p-1(\bmod 2 p-2)$ if $k$ is even. Thus it suffices to show that

$$
\psi_{\wedge}^{p^{k}}\left(J_{r}\right)=-r \beta^{r+p-p^{k}} J_{p-1}+\alpha_{r} J_{p}
$$

By (4.1), $\phi_{\beta}\left(\psi_{\wedge}^{p^{k}}\left(J_{r}\right)\right)=\alpha_{r}$, just as for $r$ odd. Also, by Lemma 3.4,

$$
\phi_{\beta}\left(-r \beta^{r+p-p^{k}} J_{p-1}+\alpha_{r} J_{p}\right)=-r \bar{\alpha}+\alpha_{r}(1+\bar{\alpha})=-r \bar{\alpha}+\alpha_{r}+r \bar{\alpha}=\alpha_{r} .
$$


For $\gamma \in M_{2 p}^{*},(4.1)$ gives

$$
\phi_{\gamma}\left(\psi_{\wedge}^{p^{k}}\left(J_{r}\right)\right)=\beta^{(r-1) p^{k}} \sum_{j=0}^{r-1} \gamma^{(r-1-2 j) p^{k}}=r \beta^{(r-1) p^{k}} \gamma^{p^{k}}=r \beta^{r-p^{k}} \gamma^{p} .
$$

Also, by Lemma 3.4,

$$
\phi_{\gamma}\left(-r \beta^{r+p-p^{k}} J_{p-1}+\alpha_{r} J_{p}\right)=r \beta^{r+p-p^{k}} \gamma^{p} \beta^{p-2}=r \beta^{r-p^{k}} \gamma^{p} .
$$

Thus the result again follows by Corollary 3.2 .

LEMMA 4.3. Let $n$ be a positive integer and $r \in\{1, \ldots, p\}$. Then

$$
\psi_{S}^{n}\left(J_{r}\right)-\psi_{\wedge}^{n}\left(J_{r}\right)=\left\{\begin{array}{lll}
0 & \text { if } n \neq 00 & (\bmod p) ; \\
p\left(J_{1}-W^{(r-1) n / p}\right) & \text { if } n \equiv 0 & (\bmod p) .
\end{array}\right.
$$

PROOF. By (2.4) and Theorem 3.5,

$$
\begin{aligned}
\psi_{S}^{1}\left(J_{r}\right) & +\psi_{S}^{2}\left(J_{r}\right) t+\cdots \\
= & \frac{d}{d t} \log \left(1-W^{r-1} t^{p}\right)-\frac{d}{d t} \log \left(1-t^{p}\right)-\frac{d}{d t} \log \wedge\left(J_{r},-t\right) .
\end{aligned}
$$

Hence, by (2.3) and multiplication by $t$,

$$
\begin{aligned}
& \left(\psi_{S}^{1}\left(J_{r}\right)-\psi_{\wedge}^{1}\left(J_{r}\right)\right) t+\left(\psi_{S}^{2}\left(J_{r}\right)-\psi_{\wedge}^{2}\left(J_{r}\right)\right) t^{2}+\cdots \\
& \quad=-p W^{r-1} t^{p}\left(1-W^{r-1} t^{p}\right)^{-1}+p t^{p}\left(1-t^{p}\right)^{-1}
\end{aligned}
$$

The result follows by comparing coefficients.

THEOREM 4.4. Let $k$ be a positive integer and let $r \in\{1, \ldots, p\}$. If $r$ is odd,

$$
\psi_{S}^{p^{k}}\left(J_{r}\right)=\left(p-(p-r) \beta^{r-1}\right)\left(J_{1}-J_{p}\right)+\alpha_{r} J_{p}
$$

If $r$ is even,

$$
\psi_{S}^{p^{k}}\left(J_{r}\right)= \begin{cases}p\left(J_{1}-J_{p}\right)+(p-r) \beta^{r} J_{p-1}+\alpha_{r} J_{p} & \text { if } k \text { is odd } \\ p\left(J_{1}-J_{p}\right)+(p-r) \beta^{r+p-1} J_{p-1}+\alpha_{r} J_{p} & \text { if } k \text { is even }\end{cases}
$$

PROOF. This holds for both $p$ odd and $p=2$. It follows by straightforward calculations from Lemma 4.3, Theorem 4.2 and Lemma 4.1.

LEMMA 4.5. For all $k$, $i$ and $r, \psi_{S}^{p^{k}}\left(\alpha^{i} J_{r}\right)=\alpha^{i} \psi_{S}^{p^{k}}\left(J_{r}\right)$.

PROOF. This follows from Lemma 2.5, since $\alpha^{i p^{k}}=\alpha^{i}$. 
The following lemma is proved by direct calculation from Theorem 4.4 and Lemma 4.5, using the linearity of $\psi_{S}^{p}$ and $\psi_{S}^{p^{2}}$.

LEMMA 4.6. Let $r \in\{1, \ldots, p\}$. If $r$ is odd,

$$
\begin{aligned}
\left(\psi_{S}^{p} \circ \psi_{S}^{p}\right)\left(J_{r}\right) & =\left(\psi_{S}^{p^{2}} \circ \psi_{S}^{p^{2}}\right)\left(J_{r}\right)=\left(\psi_{S}^{p^{2}} \circ \psi_{S}^{p}\right)\left(J_{r}\right)=\left(\psi_{S}^{p} \circ \psi_{S}^{p^{2}}\right)\left(J_{r}\right) \\
& =\left(p-p^{2}+(p-1)(p-r) \beta^{r-1}+p \alpha_{r}\right)\left(J_{1}-J_{p}\right)+\alpha_{r} J_{p} .
\end{aligned}
$$

If $r$ is even,

$$
\begin{aligned}
\left(\psi_{S}^{p} \circ \psi_{S}^{p}\right)\left(J_{r}\right)= & p\left(1-p+(p-r) \beta^{r}+\alpha_{r}\right)\left(J_{1}-J_{p}\right) \\
& +(p-r) \beta^{r+p-1} J_{p-1}+\alpha_{r} J_{p}, \\
\left(\psi_{S}^{p^{2}} \circ \psi_{S}^{p^{2}}\right)\left(J_{r}\right)= & p\left(1-p+(p-r) \beta^{r+p-1}+\alpha_{r}\right)\left(J_{1}-J_{p}\right) \\
& +(p-r) \beta^{r+p-1} J_{p-1}+\alpha_{r} J_{p}, \\
\left(\psi_{S}^{p^{2}} \circ \psi_{S}^{p}\right)\left(J_{r}\right)= & p\left(1-p+(p-r) \beta^{r}+\alpha_{r}\right)\left(J_{1}-J_{p}\right) \\
& +(p-r) \beta^{r} J_{p-1}+\alpha_{r} J_{p}, \\
\left(\psi_{S}^{p} \circ \psi_{S}^{p^{2}}\right)\left(J_{r}\right)= & p\left(1-p+(p-r) \beta^{r+p-1}+\alpha_{r}\right)\left(J_{1}-J_{p}\right) \\
& +(p-r) \beta^{r} J_{p-1}+\alpha_{r} J_{p} .
\end{aligned}
$$

The remaining lemma of this section follows easily from Theorem 4.4 and Lemma 4.6. It is required for the calculations in Section 5.

LEMMA 4.7. Let $r \in\{1, \ldots, p\}$. If $r$ is odd,

$$
\begin{aligned}
& \left(-\psi_{S}^{p}+\psi_{S}^{p^{2}} \circ \psi_{S}^{p}+p \psi_{S}^{p^{2}}\right)\left(J_{r}\right)=\left(-\psi_{S}^{p^{2}}+\psi_{S}^{p} \circ \psi_{S}^{p}+p \psi_{S}^{p}\right)\left(J_{r}\right)=p \alpha_{r} J_{1} \\
& \left(-\psi_{S}^{p^{2}}+\psi_{S}^{p^{2}} \circ \psi_{S}^{p^{2}}+p \psi_{S}^{p}\right)\left(J_{r}\right)=\left(-\psi_{S}^{p}+\psi_{S}^{p} \circ \psi_{S}^{p^{2}}+p \psi_{S}^{p^{2}}\right)\left(J_{r}\right)=p \alpha_{r} J_{1} .
\end{aligned}
$$

If $r$ is even,

$$
\begin{aligned}
\left(-\psi_{S}^{p}+\psi_{S}^{p^{2}} \circ \psi_{S}^{p}+p \psi_{S}^{p^{2}}\right)\left(J_{r}\right) & =p(p-r) \beta^{r}\left(J_{1}+\beta^{p-1} J_{p-1}-J_{p}\right)+p \alpha_{r} J_{1}, \\
\left(-\psi_{S}^{p^{2}}+\psi_{S}^{p} \circ \psi_{S}^{p}+p \psi_{S}^{p}\right)\left(J_{r}\right) & =p(p-r) \beta^{r}\left(J_{1}+J_{p-1}-J_{p}\right)+p \alpha_{r} J_{1}, \\
\left(-\psi_{S}^{p^{2}}+\psi_{S}^{p^{2}} \circ \psi_{S}^{p^{2}}+p \psi_{S}^{p}\right)\left(J_{r}\right) & =p(p-r) \beta^{r+p-1}\left(J_{1}+\beta^{p-1} J_{p-1}-J_{p}\right)+p \alpha_{r} J_{1}, \\
\left(-\psi_{S}^{p}+\psi_{S}^{p} \circ \psi_{S}^{p^{2}}+p \psi_{S}^{p^{2}}\right)\left(J_{r}\right) & =p(p-r) \beta^{r+p-1}\left(J_{1}+J_{p-1}-J_{p}\right)+p \alpha_{r} J_{1} .
\end{aligned}
$$

\section{The key special case}

Let $K$ be a field of prime characteristic $p$, and let $Q$ be a group of order $p(p-1)$ generated by elements $a$ and $b$ with relations $a^{p}=1, b^{p-1}=1$ and $b^{-1} a b=a^{l}$, 
where $l$ is a positive integer such that the image of $l$ in $K$ has multiplicative order $p-1$. In other words, $Q$ is isomorphic to the holomorph of a group of order $p$. In this section we shall prove Theorem 1.1 for $Q$ by proving the following result.

THEOREM 5.1. Let $K$ be a field of prime characteristic $p$ and let $Q$ be isomorphic to the holomorph of a group of order $p$. Then $\Phi_{K Q}^{n}=\zeta_{K Q}^{n}$ for all $n$.

The $K Q$-modules $J_{1}, \ldots, J_{p}$ and $\alpha$ are defined as in Section 3. When convenient we also use $\beta$ such that $\beta^{2}=\alpha$, as in Section 3. There are, up to isomorphism, precisely $p(p-1)$ indecomposable $K Q$-modules. In [6, Section 4$]$ these were denoted by $J_{i, r}$, for $i=0, \ldots, p-2$ and $r=1, \ldots, p$, and further details can be found there. It is easily checked that, in the notation of the present paper, $J_{i, r}=\alpha^{i} J_{r}$.

By [6, Theorem 4.4] with $i=0$, combined with [6, Lemma 4.1], we have

$$
\sum_{d \mid n}\left(\Phi_{K Q}^{d} \circ \psi_{S}^{n / d}\right)\left(J_{r}\right)= \begin{cases}J_{r} & \text { for } n=1 \\ -p\left(J_{p}-\alpha J_{p-1}-J_{1}\right) & \text { for } n=p \\ 0 & \text { for } n \neq 1, p\end{cases}
$$

for $r=2, \ldots, p$. Also, by Lemma 2.5 ,

$$
\begin{aligned}
\Phi_{K Q}^{n}\left(J_{1}\right) & =\mu(n) J_{1}, & & \text { for all } n, \quad \text { and } \\
\Phi_{K Q}^{n}\left(\alpha^{i} J_{r}\right) & =\alpha^{n i} \Phi_{K Q}^{n}\left(J_{r}\right), & & \text { for all } n, i \text { and } r .
\end{aligned}
$$

Equations (5.2)-(5.3) yield $\Phi_{K Q}^{n}\left(\alpha^{i} J_{1}\right)$ for all $n$ and all $i$. For $r \geq 2,(5.1)$ and (5.3) yield $\Phi_{K Q}^{n}\left(\alpha^{i} J_{r}\right)$ in terms of Adams operations and values of the functions $\Phi_{K Q}^{d}$ for proper divisors $d$ of $n$. Thus $\Phi_{K Q}^{1}, \Phi_{K Q}^{2}, \ldots$ are the unique linear functions on $R_{K Q}$ satisfying (5.1)-(5.3).

LEMMA 5.2. If $n=p^{k} m$ where $p \nmid m$, then $\Phi_{K Q}^{n}=\Phi_{K Q}^{p^{k}} \circ \mu(m) \psi_{S}^{m}$.

Proof. By [6, Theorem 4.4, Lemma 4.6 and Lemma 5.1 (ii)], we have $\Phi_{K Q}^{n}=$ $\Phi_{K Q}^{p^{k}} \circ \Phi_{K Q}^{m}$. The result follows by (1.3).

By (5.1) with $n=p, \psi_{S}^{p}\left(J_{r}\right)+\Phi_{K Q}^{p}\left(J_{r}\right)=-p\left(J_{p}-\alpha J_{p-1}-J_{1}\right)$, for all $r \geq 2$. However, $\zeta_{K Q}^{p}=\Phi_{K Q}^{p}$, by the definition of $\zeta_{K Q}^{p}$. Thus, for all $r \geq 2$,

$$
\zeta_{K Q}^{p}\left(J_{r}\right)=p J_{1}+p \alpha J_{p-1}-p J_{p}-\psi_{S}^{p}\left(J_{r}\right)
$$

Also, by Lemma 2.5 ,

$$
\zeta_{K Q}^{p}\left(J_{1}\right)=-J_{1}
$$


From the definition of $\zeta_{K Q}^{n}$, if $n=p^{k} m$ where $p \nmid m$, then

$$
\zeta_{K Q}^{n}=\zeta_{K Q}^{p^{k}} \circ \mu(m) \psi_{S}^{m}
$$

The following result is easily obtained from (5.5), (5.4) and Theorem 4.4. (Recall that $\beta^{2}=\alpha$ and $\bar{\alpha}=1+\alpha+\cdots+\alpha^{p-2}$.)

LEMMA 5.3. We have $\zeta_{K Q}^{p}\left(J_{1}\right)=-J_{1}$ and $\zeta_{K Q}^{p}\left(J_{p}\right)=p \alpha J_{p-1}-(1+\bar{\alpha}) J_{p}$. Also, for $p$ odd, $\zeta_{K Q}^{p}\left(J_{p-1}\right)=\left(p \alpha-\beta^{p-1}\right) J_{p-1}-\bar{\alpha} J_{p}$.

Since $R_{K Q}$ is spanned by the modules $\alpha^{i} J_{r}$, Theorem 4.4 and Lemma 4.5 give

$$
\psi_{S}^{p}=\psi_{S}^{p^{3}}=\psi_{S}^{p^{5}}=\cdots \quad \text { and } \quad \psi_{S}^{p^{2}}=\psi_{S}^{p^{4}}=\psi_{S}^{p^{6}}=\cdots \quad \text { on } R_{K Q}
$$

LEMMA 5.4. Let $m$ be a positive integer, where $m \geq 3$. Then

$$
-\psi_{S}^{p^{m}}+\psi_{S}^{p^{2}} \circ \psi_{S}^{p^{m-2}}+p \psi_{S}^{p^{m-1}}+\zeta_{K \mathcal{Q}}^{p} \circ\left(-\psi_{S}^{p^{m-1}}+\psi_{S}^{p} \circ \psi_{S}^{p^{m-2}}+p \psi_{S}^{p^{m-2}}\right)=0
$$

PROOF. Let $\chi$ and $\chi^{\prime}$ be the linear functions on $R_{K Q}$ defined by

$$
\begin{aligned}
& \chi=-\psi_{S}^{p}+\psi_{S}^{p^{2}} \circ \psi_{S}^{p}+p \psi_{S}^{p^{2}}+\zeta_{K Q}^{p} \circ\left(-\psi_{S}^{p^{2}}+\psi_{S}^{p} \circ \psi_{S}^{p}+p \psi_{S}^{p}\right), \\
& \chi^{\prime}=-\psi_{S}^{p^{2}}+\psi_{S}^{p^{2}} \circ \psi_{S}^{p^{2}}+p \psi_{S}^{p}+\zeta_{K Q}^{p} \circ\left(-\psi_{S}^{p}+\psi_{S}^{p} \circ \psi_{S}^{p^{2}}+p \psi_{S}^{p^{2}}\right) .
\end{aligned}
$$

By (5.7), it suffices to prove that $\chi=\chi^{\prime}=0$. By Lemma 4.5, $\psi_{S}^{p^{k}}\left(\alpha^{i} J_{r}\right)=\alpha^{i} \psi_{S}^{p^{k}}\left(J_{r}\right)$ for all $k, i$ and $r$. Similarly, by Lemma $2.5, \zeta_{K Q}^{p}\left(\alpha^{i} J_{r}\right)=\alpha^{i} \zeta_{K Q}^{p}\left(J_{r}\right)$. Hence it suffices to show that $\chi\left(J_{r}\right)=\chi^{\prime}\left(J_{r}\right)=0$ for all $r$. This follows by direct calculation from Lemmas 4.7 and 5.3.

COROLLARY 5.5. For all $k \geq 3, \zeta_{K Q}^{p^{k}}=p \zeta_{K Q}^{p^{k-1}}$

PROOF. By (5.7) and the definition of $\zeta_{K Q}^{p^{k}}$, we have $\zeta_{K Q}^{p^{k}}=p^{2} \zeta_{K Q}^{p^{k-2}}$ for all $k \geq 4$. Thus it suffices to prove that $\zeta_{K Q}^{p^{3}}=p \zeta_{K Q}^{p^{2}}$. However,

$$
\begin{aligned}
\zeta_{K Q}^{p^{3}}-p \zeta_{K Q}^{p^{2}} & =-\psi_{S}^{p^{3}}-\zeta_{K Q}^{p} \circ \psi_{S}^{p^{2}}-\zeta_{K Q}^{p^{2}} \circ \psi_{S}^{p}-p \zeta_{K Q}^{p^{2}} \\
& =-\psi_{S}^{p^{3}}-\zeta_{K Q}^{p} \circ \psi_{S}^{p^{2}}+\left(\psi_{S}^{p^{2}}+\zeta_{K Q}^{p} \circ \psi_{S}^{p}\right) \circ \psi_{S}^{p}+p\left(\psi_{S}^{p^{2}}+\zeta_{K Q}^{p} \circ \psi_{S}^{p}\right) \\
& =-\psi_{S}^{p^{3}}+\psi_{S}^{p^{2}} \circ \psi_{S}^{p}+p \psi_{S}^{p^{2}}+\zeta_{K Q}^{p} \circ\left(-\psi_{S}^{p^{2}}+\psi_{S}^{p} \circ \psi_{S}^{p}+p \psi_{S}^{p}\right) .
\end{aligned}
$$

This is equal to 0 , by Lemma 5.4. Therefore $\zeta_{K_{Q}}^{p^{3}}=p \zeta_{K Q}^{p^{2}}$.

LEMMA 5.6. For $k \geq 2, \sum_{j=0}^{k} \zeta_{K Q^{p^{j}}} \circ \psi_{S}^{p^{k-j}}=0$. 
PROOF. For $k=2$, the result follows from the definition of $\zeta_{K Q}^{p^{2}}$. Suppose that $m \geq 3$ and that the result holds for $k=m-1$. Then, by Corollary 5.5,

$$
\begin{aligned}
\sum_{j=0}^{m} \zeta_{K Q}^{p^{j}} \circ \psi_{S}^{p^{m-j}} & =\psi_{S}^{p^{m}}+\zeta_{K Q}^{p} \circ \psi_{S}^{p^{m-1}}+\zeta_{K Q}^{p^{2}} \circ \psi_{S}^{p^{m-2}}+\sum_{j=3}^{m} \zeta_{K Q}^{p^{j}} \circ \psi_{S}^{p^{m-j}} \\
& =\psi_{S}^{p^{m}}+\zeta_{K Q}^{p} \circ \psi_{S}^{p^{m-1}}+\zeta_{K Q}^{p^{2}} \circ \psi_{S}^{p^{m-2}}+p \sum_{j=2}^{m-1} \zeta_{K Q}^{p^{j}} \circ \psi_{S}^{p^{m-1-j}} \\
& =\psi_{S}^{p^{m}}+\zeta_{K Q}^{p} \circ \psi_{S}^{p^{m-1}}+\zeta_{K Q}^{p^{2}} \circ \psi_{S}^{p^{m-2}}-p\left(\psi_{S}^{p^{m-1}}+\zeta_{K Q}^{p} \circ \psi_{S}^{p^{m-2}}\right) .
\end{aligned}
$$

By definition, $\zeta_{K Q}^{p^{2}}=-\left(\psi_{S}^{p^{2}}+\zeta_{K Q}^{p} \circ \psi_{S}^{p}\right)$. Therefore $\sum_{j=0}^{m} \zeta_{K Q}^{p^{j}} \circ \psi_{S}^{p^{m-j}}$ is equal to

$$
-\left(-\psi_{S}^{p^{m}}+\psi_{S}^{p^{2}} \circ \psi_{S}^{p^{m-2}}+p \psi_{S}^{p^{m-1}}+\zeta_{K Q}^{p} \circ\left(-\psi_{S}^{p^{m-1}}+\psi_{S}^{p} \circ \psi_{S}^{p^{m-2}}+p \psi_{S}^{p^{m-2}}\right)\right) .
$$

This is equal to 0 , by Lemma 5.4. Hence the result holds for $k=m$. By induction, the result holds for all $k \geq 2$.

PROOF OF THEOREM 5.1. We need to prove that $\Phi_{K Q}^{n}=\zeta_{K Q}^{n}$ for all $n$. By (5.6) and Lemma 5.2, it suffices to prove that $\Phi_{K Q}^{p^{k}}=\zeta_{K Q}^{p^{k}}$ for all $k \geq 0$. We consider (5.1)-(5.3) restricted to values of $n$ which are powers of $p$. These equations uniquely determine the linear functions $\Phi_{K Q}^{1}, \Phi_{K Q}^{p}, \Phi_{K Q}^{p^{2}}, \ldots$ Hence it suffices to show that the functions $\zeta_{K Q}^{1}, \zeta_{K Q}^{p}, \zeta_{K Q}^{p^{2}}, \ldots$ satisfy the same equations. Equations (5.2) and (5.3) for the $\zeta_{K Q}^{p^{k}}$ are given by Lemma 2.5. This leaves (5.1). For $n=1$ the required result is clear. For $n=p$ it is given by (5.4). Finally, for $n=p^{k}$ with $k \geq 2$, the result is given by Lemma 5.6.

\section{Normal Sylow subgroup}

In this section we prove Theorem 1.1 for the case in which the Sylow $p$-subgroup of $G$ has order $p$ and is normal. It suffices to prove the following result.

THEOREM 6.1. Let $K$ be a field of prime characteristic $p$ and let $G$ be a finite group with a normal Sylow p-subgroup of order $p$. Then $\Phi_{K G}^{n}=\zeta_{K G}^{n}$ for all $n$.

We use the notation of Section 3. In particular, $G=H P$, where $P$ is the Sylow $p$-subgroup of $G$ and $H$ is a $p^{\prime}$-group. We consider the $K G$-modules $J_{1}, \ldots, J_{p}$ and $\alpha$. When convenient we also use $\widehat{K}, \widetilde{G}, \beta$ and $\lambda$, as in Section 3 .

LEMMA 6.2. The isomorphism classes of finite-dimensional indecomposable $K G$ modules are represented by the modules $I \otimes J_{r}$, where $1 \leq r \leq p$ and $I$ ranges over 
a set of representatives of the isomorphism classes of irreducible $K H$-modules, these being regarded as $K G$-modules through the projection $G \rightarrow H$.

Proof. This is given by [14, Proposition 1.1], where it is not necessary to assume that the field is a splitting field. See also [16, Proposition 4.4].

LEMMA 6.3. Let $U$ and $V$ be elements of $R_{K G}$ such that $U \downarrow_{H_{0} P}=V \downarrow_{H_{0} P}$ for every cyclic subgroup $H_{0}$ of $H$. Then $U=V$.

Proof. This is given by [16, Corollary 4.4]. It can be obtained by applying Lemma 6.2 to $G$ and to the subgroups $H_{0} P$.

LEMMA 6.4. Let $U$ be a finite-dimensional $K H$-module, regarded as a $K G$-module. Then, for $r=1, \ldots, p$ and every positive integer $n$,

$$
\begin{aligned}
\psi_{\wedge}^{n}\left(U J_{r}\right) & =\psi_{\lambda}^{n}(U) \psi_{\wedge}^{n}\left(J_{r}\right), & \psi_{S}^{n}\left(U J_{r}\right) & =\psi_{S}^{n}(U) \psi_{S}^{n}\left(J_{r}\right), \\
\Phi_{K G}^{n}\left(U J_{r}\right) & =\psi_{\wedge}^{n}(U) \Phi_{K G}^{n}\left(J_{r}\right), & \zeta_{K G}^{n}\left(U J_{r}\right) & =\psi_{\wedge}^{n}(U) \zeta_{K G}^{n}\left(J_{r}\right) .
\end{aligned}
$$

PROOF. By Lemma 2.4, we may assume that $K$ is algebraically closed. By Lemmas 6.3 and 2.3 it suffices to prove the corresponding results for the subgroups $H_{0} P$, where $H_{0}$ is a cyclic subgroup of $H$. Thus we may assume that $H$ is cyclic. Therefore $U$ is isomorphic to the direct sum of one-dimensional modules, and it suffices to consider the case where $U$ is one-dimensional. Let $\psi^{n}$ denote either $\psi_{\wedge}^{n}, \psi_{S}^{n}, \Phi_{K G}^{n}$ or $\zeta_{K G}^{n}$. Thus, by Lemma $2.5, \psi^{n}\left(U J_{r}\right)=U^{n} \psi^{n}\left(J_{r}\right)$ and $U^{n}=\psi_{\wedge}^{n}(U)=\psi_{S}^{n}(U)$. The result follows.

LEMMA 6.5. For $r=1, \ldots, p$ and all $n, \Phi_{K G}^{n}\left(J_{r}\right)=\zeta_{K G}^{n}\left(J_{r}\right)$.

Proof. Let $Q$ be the holomorph of $P$, identified with the group $Q$ of Section 5 . Thus $Q=\operatorname{Aut}(P) P$ where $P$ is generated by $a$ and $\operatorname{Aut}(P)$ is generated by $b$. The action of $H$ on $P$ by conjugation gives a homomorphism $H \rightarrow \operatorname{Aut}(P)$. This extends to a homomorphism $\tau: G \rightarrow Q$ which is the identity on $P$ and gives a homomorphism $\tau^{*}: R_{K Q} \rightarrow R_{K G}$. It is easy to check that $\tau^{*}\left(J_{r}\right)=J_{r}$ (using the same notation $J_{r}$ in connection with both $Q$ and $G$ ). By Theorem 5.1, $\Phi_{K Q}^{n}\left(J_{r}\right)=\zeta_{K Q}^{n}\left(J_{r}\right)$. Hence $\tau^{*}\left(\Phi_{K Q}^{n}\left(J_{r}\right)\right)=\tau^{*}\left(\zeta_{K Q}^{n}\left(J_{r}\right)\right)$. Therefore $\Phi_{K G}^{n}\left(J_{r}\right)=\zeta_{K G}^{n}\left(J_{r}\right)$, by Lemma 2.3.

PROOF OF THEOREM 6.1. By Lemma 6.2, it suffices to show that we have

$$
\Phi_{K G}^{n}\left(I J_{r}\right)=\zeta_{K G}^{n}\left(I J_{r}\right)
$$

for $r=1, \ldots, p$ and all irreducible $K H$-modules $I$. However, by Lemma 6.4, $\Phi_{K G}^{n}\left(I J_{r}\right)=\psi_{\Lambda}^{n}(I) \Phi_{K G}^{n}\left(J_{r}\right)$ and $\zeta_{K G}^{n}\left(I J_{r}\right)=\psi_{\Lambda}^{n}(I) \zeta_{K G}^{n}\left(J_{r}\right)$. Thus the result follows from Lemma 6.5 . 
If we wish to apply Theorem 1.1 for our group $G$ with a normal Sylow $p$-subgroup we need to know the Adams operations on $R_{K G}$ and the functions $\zeta_{K G}^{p^{k}}$ (or, at least, $\zeta_{K G}^{p}$ ). By Lemmas 6.2 and 6.4, these can be obtained from the Adams operations on $R_{K H}$ and the values of the Adams operations and the functions $\zeta_{K G}^{p^{k}}$ on the modules $J_{r}$. These values of $\zeta_{K G}^{p^{k}}$ are given by the following result, in the notation of Section 3 . (Recall that $\beta^{2}=\alpha$ and $\alpha_{r}=1+\alpha+\cdots+\alpha^{r-1}$.)

$$
\begin{aligned}
& \text { LEMMA 6.6. We have } \zeta_{K G}^{p}\left(J_{1}\right)=-J_{1} \text { and } \zeta_{K G}^{p^{2}}\left(J_{1}\right)=0 \text {. For } r \geq 2, \\
& \qquad \zeta_{K G}^{p}\left(J_{r}\right)= \begin{cases}p \alpha J_{p-1}+(p-r) \beta^{r-1}\left(J_{1}-J_{p}\right)-\alpha_{r} J_{p} & \text { if } r \text { is odd } \\
p \alpha J_{p-1}-(p-r) \beta^{r} J_{p-1}-\alpha_{r} J_{p} & \text { if } r \text { is even, }\end{cases} \\
& \zeta_{K G}^{p^{2}}\left(J_{r}\right)= \begin{cases}p \alpha\left(p-(p-r) \beta^{r-1}-\alpha_{r}\right) J_{p-1} & \text { if } r \text { is odd } ; \\
p \alpha\left(p-(p-r) \beta^{r}-\alpha_{r}\right) J_{p-1} & \text { if } r \text { is even. }\end{cases}
\end{aligned}
$$

Furthermore, $\zeta_{K G}^{p^{k}}\left(J_{r}\right)=p \zeta_{K G}^{p^{k-1}}\left(J_{r}\right)$ for all $r$ and $k \geq 3$.

Proof. We use the homomorphism $\tau^{*}: R_{K Q} \rightarrow R_{K G}$, as in the proof of Lemma 6.5. As observed there, $\tau^{*}\left(J_{r}\right)=J_{r}$. It is also easy to verify that $\tau^{*}(\alpha)=\alpha$ (using the same notation $\alpha$ in connection with both $Q$ and $G$ ). The powers of $\beta$ in the formulae of the lemma are actually powers of $\alpha$, since $\beta^{2}=\alpha$. Thus, by Lemma 2.3, it suffices to prove these formulae for $Q$ instead of $G$. The results for $\zeta_{K Q}^{p}$ are obtained by straightforward calculations from (5.4), (5.5) and Theorem 4.4. Also, by definition, $\zeta_{K Q}^{p^{2}}\left(J_{r}\right)=-\psi_{S}^{p^{2}}\left(J_{r}\right)-\zeta_{K Q}^{p}\left(\psi_{S}^{p}\left(J_{r}\right)\right)$. This allows the calculation of $\zeta_{K Q}^{p^{2}}$. The last statement of the lemma is given by Corollary 5.5.

As far as Adams operations on $R_{K G}$ are concerned, we only need finitely many because of the periodicity given by the following result.

LEMMA 6.7. Let $q=\left|H / C_{H}(P)\right|$ and let e be the least common multiple of $2 p q$ and the orders of the elements of $H$. Then, for all $n, \psi_{\wedge}^{n}=\psi_{\wedge}^{n+e}$ and $\psi_{S}^{n}=\psi_{S}^{n+e}$.

PROOF. This was proved in [16, Proposition 4.7], using results for GL(2, $p)$. We sketch an independent proof.

By Lemma 6.2 it suffices to show that we have $\psi_{\wedge}^{n}\left(I J_{r}\right)=\psi_{\wedge}^{n+e}\left(I J_{r}\right)$ and $\psi_{S}^{n}\left(I J_{r}\right)=\psi_{S}^{n+e}\left(I J_{r}\right)$ for $r=1, \ldots, p$ and all irreducible $K H$-modules $I$. By Lemma 2.6 and the choice of $e$, the elements $\psi_{\wedge}^{n}(I), \psi_{\wedge}^{n+e}(I), \psi_{S}^{n}(I)$ and $\psi_{S}^{n+e}(I)$ of $R_{K H}$ have the same Brauer character. Thus they are equal, by Lemma 2.9. Therefore, by Lemma 6.4, it suffices to prove that $\psi_{\Lambda}^{n}\left(J_{r}\right)=\psi_{\Lambda}^{n+e}\left(J_{r}\right)$ and $\psi_{S}^{n}\left(J_{r}\right)=\psi_{S}^{n+e}\left(J_{r}\right)$. In fact we prove the stronger result that, for all $n, \psi_{\wedge}^{n}\left(J_{r}\right)=\psi_{\wedge}^{n+2 p q}\left(J_{r}\right)$ and 
$\psi_{S}^{n}\left(J_{r}\right)=\psi_{s}^{n+2 p q}\left(J_{r}\right)$. For this we may assume that $K=\widehat{K}$ and $G=\widetilde{G}$, in the notation of Section 3. By (4.1),

$$
\psi_{\wedge}^{n}\left(J_{r}\right)=\beta^{(r-1) n} \sum_{j=0}^{r-1} \lambda^{(r-1-2 j) n}, \quad \psi_{\wedge}^{n+2 p q}\left(J_{r}\right)=\beta^{(r-1)(n+2 p q)} \sum_{j=0}^{r-1} \lambda^{(r-1-2 j)(n+2 p q)} .
$$

However, $\beta^{(r-1) n}=\beta^{(r-1)(n+2 p q)}$, since $\beta^{2 q}=1$. Also, from the formula for $J_{p}$ given by (3.1), $\lambda^{2 p}-1=\left(\lambda^{2}-1\right) \lambda^{p-1} \beta^{-p+1} J_{p} \in \Omega$, where $\Omega$ is the ideal of $R_{K G}[\lambda]$ generated by $J_{p}$. Therefore $\psi_{\wedge}^{n+2 p q}\left(J_{r}\right)=\psi_{\wedge}^{n}\left(J_{r}\right)+U$, where $U \in \Omega \cap R_{K G}$. However, $\Omega \cap R_{K G}=R_{K G} J_{p}$. Thus $U \in\left(R_{K G}\right)_{\mathrm{proj}}$, in the notation at the end of Section 2. Also, by Lemma 2.6, $\operatorname{Br}\left(\psi_{\wedge}^{n+2 p q}\left(J_{r}\right)\right)=\operatorname{Br}\left(\psi_{\wedge}^{n}\left(J_{r}\right)\right)$. Thus $\psi_{\wedge}^{n+2 p q}\left(J_{r}\right)=\psi_{\wedge}^{n}\left(J_{r}\right)$ by Lemma 2.9. From this we obtain $\psi_{S}^{n+2 p q}\left(J_{r}\right)=\psi_{S}^{n}\left(J_{r}\right)$ by Lemmas 4.3 and 4.1 .

The values of the Adams operations on the $J_{r}$ can, at least in principle, be calculated using (4.1) and Lemma 4.3. (See [1] for corresponding calculations for the group of order $p$.)

\section{The general case}

Let $K$ be a field of prime characteristic $p$. If $G$ is a finite $p^{\prime}$-group then $\Phi_{K G}^{n}=\zeta_{K G}^{n}$ for all $n$, by Lemmas 2.8 and 2.9. (Indeed, we also have $\Phi_{K G}^{n}=\mu(n) \psi_{S}^{n}$ by Lemmas 2.6 and 2.8). Thus, to complete the proof of Theorem 1.1, we only need consider the case where $G$ is a finite group with a Sylow $p$-subgroup $P$ of order $p$. We write $N$ for the normalizer of $P$ in $G$. Thus $N$ is a finite group with a normal Sylow $p$-subgroup of order $p$, and the results of Sections 3-6 apply (with $N$ replacing $G$ ). We write $N=H P$, where $H$ is a $p^{\prime}$-group.

The subgroup $P$ of $G$ is a trivial-intersection set, so a simple form of the Green correspondence applies (see [2, Theorem 10.1], where the field does not need to be algebraically closed): there is a one-one correspondence between finite-dimensional non-projective indecomposable $K G$-modules and finite-dimensional non-projective indecomposable $K N$-modules. Here, if $V$ corresponds to $V^{*}$ then $V \downarrow_{N}$ is the direct sum of $V^{*}$ and a projective module. It follows that if $V, V^{\prime} \in R_{K G}$ and $V \downarrow_{N}=V^{\prime} \downarrow_{N}$ then $V_{\text {nonp }}=V_{\text {nonp }}^{\prime}$. The proof of Theorem 1.1 is completed by the following result.

THEOREM 7.1. Let $K$ be a field of prime characteristic $p$ and let $G$ be a finite group with a Sylow p-subgroup of order $p$. Then $\Phi_{K G}^{n}=\zeta_{K G}^{n}$ for all $n$.

Proof. Let $V$ be a finite-dimensional $K G$-module. Then, by Theorem 6.1 and Lemma 2.3, $\Phi_{K G}^{n}(V) \downarrow_{N}=\zeta_{K G}^{n}(V) \downarrow_{N}$. Hence, by the Green correspondence, $\Phi_{K G}^{n}(V)_{\text {nonp }}=\zeta_{K G}^{n}(V)_{\text {nonp }}$. However, $\operatorname{Br}\left(\Phi_{K G}^{n}(V)\right)=\operatorname{Br}\left(\zeta_{K G}^{n}(V)\right)$, by Lemma 2.8 . Therefore $\Phi_{K G}^{n}(V)=\zeta_{K G}^{n}(V)$, by Lemma 2.9. This gives the required result. 
By Theorem 1.1 we can calculate all Lie powers $L^{n}(V)$ if we can find tensor powers, Adams operations and the $p$ th Lie powers of all indecomposables. By the next result, only finitely many Adams operations need to be found. With $H$ as defined above, let $q=\left|H / C_{H}(P)\right|$ and let $e$ be the least common multiple of $2 p q$ and the orders of the $p^{\prime}$-elements of $G$.

THEOREM 7.2. Let $K$ be a field of prime characteristic $p$ and let $G$ be a finite group with a Sylow p-subgroup of order $p$. Let $e$ be as defined above. Then, for every positive integer $n, \psi_{\wedge}^{n}=\psi_{\wedge}^{n+e}$ and $\psi_{S}^{n}=\psi_{S}^{n+e}$.

PROOF. (For $G=\mathrm{GL}(2, p)$, this is given by [15, Proposition 3.5].) Let $V$ be a finite-dimensional $K G$-module. Then, by Lemma 6.7, $\psi_{\lambda}^{n}(V) \downarrow_{N}=\psi_{\Lambda}^{n+e}(V) \downarrow_{N}$. Hence, by the Green correspondence, $\psi_{\Lambda}^{n}(V)_{\text {nonp }}=\psi_{\Lambda}^{n+e}(V)_{\text {nonp }}$. However, by Lemma 2.6 and the definition of $e, \operatorname{Br}\left(\psi_{\wedge}^{n}(V)\right)=\operatorname{Br}\left(\psi_{\wedge}^{n+e}(V)\right)$. Thus, by Lemma 2.9, $\psi_{\wedge}^{n}(V)=\psi_{\wedge}^{n+e}(V)$. Similarly, $\psi_{S}^{n}(V)=\psi_{S}^{n+e}(V)$. This gives the result.

If we have detailed information about the indecomposable $K G$-modules and $K N$ modules, the Green correspondence, and the Brauer characters of $G$, we can hope to find the Lie powers of a finite-dimensional $K G$-module $V$ from Lie powers of $K N$-modules as follows. Since $L^{n}(V) \downarrow_{N}=L^{n}\left(V \downarrow_{N}\right)$, by Lemma 2.2, $L^{n}(V) \downarrow_{N}$ can be calculated by the methods described at the end of Section 6 . Thus, by the Green correspondence, we can determine $L^{n}(V)_{\text {nonp }}$ and hence $\operatorname{Br}\left(L^{n}(V)_{\text {nonp }}\right)$. However, $\operatorname{Br}\left(L^{n}(V)\right)$ is given by Brandt's character formula (Lemma 2.7). Thus we can find $\operatorname{Br}\left(L^{n}(V)_{\text {proj }}\right)$. Therefore $L^{n}(V)_{\text {proj }}$ can be found, at least in principle, by the modular orthogonality relations. Hence we can find $L^{n}(V)$.

The connection between Lie powers of $K G$-modules and Lie powers of $K N$ modules was a key factor in obtaining the results of $[8,17]$ and $[10]$. The following theorem generalises one of the main qualitative results of [10]. Recall that the $(p-1)$ dimensional $K N$-module $J_{p-1}$ is as defined in Section 3.

THEOREM 7.3. Let $K$ be a field of prime characteristic $p$ and let $G$ be a finite group with a Sylow p-subgroup of order $p$. Let $V$ be a finite-dimensional $K G$-module and let $n$ be a positive integer. Then, in the notation established above, every non-projective indecomposable summand of $L^{n}(V)$ is either a summand of the nth tensor power $V^{n}$ or is the Green correspondent of a $K N$-module of the form $I \otimes J_{p-1}$, where $I$ is an irreducible $\mathrm{K} H$-module.

PROOF. We give a sketch only. Note that $L^{n}(V) \downarrow_{N}=L^{n}\left(V \downarrow_{N}\right)$ and $V^{n} \downarrow_{N}=$ $\left(V \downarrow_{N}\right)^{n}$. By the Green correspondence it suffices to show that every non-projective indecomposable summand of $L^{n}\left(V \downarrow_{N}\right)$ is either a summand of $\left(V \downarrow_{N}\right)^{n}$ or has the 
form $I \otimes J_{p-1}$, where $I$ is an irreducible $K H$-module. Thus we may assume that $G=N=H P$.

Write $n=p^{k} m$ where $p \nmid m$. By Theorem 1.1 and Corollary 1.2,

$$
L^{n}(V)=\frac{1}{p^{k}} \sum_{i=0}^{k} \zeta_{K G}^{p^{i}}\left(L^{m}\left(V^{p^{k-i}}\right)\right) .
$$

However, for $i=0, \ldots, k, L^{m}\left(V^{p^{k-i}}\right)$ is a summand of $V^{m p^{k-i}}$, since $p \nmid m$ (see, for example, [13, Section 3.1]). Hence it suffices to show, for $i \geq 0$, that if $Y$ is a finite-dimensional indecomposable $K G$-module then $\zeta_{K G}^{p^{i}}(Y)$ is a linear combination of projective $K G$-modules, summands of $Y^{p^{i}}$, and modules of the form $I \otimes J_{p-1}$, where $I$ is an irreducible $K H$-module. By Lemma 6.2, $Y \cong U \otimes J_{r}$ where $1 \leq r \leq p$ and $U$ is an irreducible $K H$-module. By Lemma $6.4, \zeta_{K G}^{p^{i}}(Y)=\psi_{\wedge}^{p^{i}}(U) \zeta_{K G}^{p^{i}}\left(J_{r}\right)$. However, by (2.2) or (2.3), $\psi_{\wedge}^{p^{i}}(U)$ is a linear combination of modules which are homomorphic images of $U^{p^{i}}$. Thus, since $H$ is a $p^{\prime}$-group, $\psi_{\wedge}^{p^{i}}(U)$ is a linear combination of summands of $U^{p^{i}}$. It therefore suffices to prove that $\zeta_{K G}^{p^{i}}\left(J_{r}\right)$ is a linear combination of projective modules, summands of $J_{r}^{p^{i}}$, and modules of the form $I \otimes J_{p-1}$. This is trivial for $i=0$ and, by Lemma 6.6, it is clear for $i \geq 2$. Suppose then that $i=1$. By Lemma 6.6, the result is clear for $r$ even, $r=1$ and $r=p$. By the same lemma, it is true for $r$ odd with $1<r<p$ provided that $\beta^{r-1} J_{1}$ is a summand of $J_{r}^{p}$. This can be proved as follows, using the notation of Section 3 .

It is sufficient to consider the case where $K=\widehat{K}$ and $G=\widetilde{G}$. Let $\Omega^{\prime}$ be the ideal of $R_{K G}[\lambda]$ generated by $p R_{K G}[\lambda]$ and $J_{p}$. Then, as in the proof of Lemma 6.7, $\lambda^{2 p}-1 \in \Omega^{\prime}$. Also, $\beta^{(r-1) p}=\beta^{r-1}$. However, by (3.1),

$$
J_{r}^{p} \equiv \beta^{(r-1) p} \sum_{j=0}^{r-1} \lambda^{(r-1-2 j) p} \quad\left(\bmod \Omega^{\prime}\right) .
$$

Hence $J_{r}^{p} \equiv r \beta^{r-1} J_{1}\left(\bmod \Omega^{\prime} \cap R_{K G}\right)$. However, $\Omega^{\prime} \cap R_{K G}=p R_{K G}+R_{K G} J_{p}$. Since $r$ is not divisible by $p$ it follows that $\beta^{r-1} J_{1}$ is a summand of $J_{r}^{p}$.

\section{References}

[1] G. Almkvist, 'Representations of $Z / p Z$ in characteristic $p$ and reciprocity theorems', J. Algebra 68 (1981), 1-27.

[2] J. L. Alperin, Local representation theory, Cambridge Stud. Adv. Math. 11 (Cambridge University Press, Cambridge, 1986).

[3] D. J. Benson, Representations and cohomology I (Cambridge University Press, Cambridge, 1995).

[4] A. J. Brandt, 'The free Lie ring and Lie representations of the full linear group', Trans. Amer. Math. Soc. 56 (1944), 528-536. 
[5] R. M. Bryant, 'Free Lie algebras and Adams operations', J. London Math. Soc. (2) 68 (2003), 355-370.

[6] — 'Modular Lie representations of groups of prime order', Math. Z. 246 (2004), 603-61 7.

[7] —_. 'Free Lie algebras and formal power series', J. Algebra 253 (2002), 167-188.

[8] R. M. Bryant, L. G. Kovács and R. Stöhr, 'Free Lie algebras as modules for symmetric groups', $J$. Austral. Math. Soc. Ser. A 67 (1999), 143-156.

[9] _ 'Lie powers of modules for groups of prime order', Proc. London Math. Soc. (3) 84 (2002), 343-374.

[10] 'Lie powers of modules for $G L(2, p)$ ', J. Algebra 260 (2003), 617-630.

[11] C. W. Curtis and I. Reiner, Representation theory of finite groups and associative algebras (WileyInterscience, New York, 1962).

[12] - Methods in representation theory, II (J. Wiley and Sons, New York, 1987).

[13] S. Donkin and K. Erdmann, 'Tilting modules, symmetric functions, and the module structure of the free Lie algebra', J. Algebra 203 (1998), 69-90.

[14] I. Hughes and G. Kemper, 'Symmetric powers of modular representations for groups with a Sylow subgroup of prime order', J. Algebra 241 (2001), 759-788.

[15] F. M. Kouwenhoven, 'The $\lambda$-structure of the Green ring of $G L\left(2, \mathbb{F}_{p}\right)$ in characteristic $p$, III', Comm. Algebra 18 (1990), 1701-1728.

[16] - 'The $\lambda$-structure of the Green ring of $G L\left(2, \mathbb{F}_{p}\right)$ in characteristic $p$, IV', Comm. Algebra 18 (1990), 1729-1747.

[17] L. G. Kovács and R. Stöhr, 'Lie powers of the natural module for $G L(2)$ ', J. Algebra 229 (2000), 435-462.

\section{School of Mathematics}

University of Manchester

PO Box 88

Manchester M60 1QD

England

e-mail: roger.bryant@manchester.ac.uk 
